\title{
La implementación de la televisión digital en el Perú: Un cambio tecnológico sin políticas públicas
}

\author{
Gerardo Arias Carbajal (Universidad de Lima) \\ Recibido: 15/01/2010 \\ Aceptado: 5/02/2010
}

RESUMEN: En este artículo se plantea que la coyuntura de la implementación de la televisión digital terrestre (TDT) es también una oportunidad para llevar a cabo un cambio en la estructura de la televisión peruana; no se trata solo de mejorar la calidad de la imagen, sino también de implementar una serie de políticas públicas que propicien un cambio real e importante en la televisión. El autor propone las siguientes líneas de políticas públicas para su discusión en el proceso de implementación de la TDT: a) ingreso de nuevos operadores, b) dinamizar la industria de contenidos, c) implementación de la TDT en televisoras regionales y locales, d) contribuir con la inclusión social mediante la interactividad, e) gratuidad de la televisión de señal abierta, independientemente del soporte, y f) condiciones de explotación de acuerdo con los modelos de negocio que cada canal pretende poner en marcha.

Palabras clave: Televisión digital terrestre (TDT) / apagón analógico / televisión nacional / políticas públicas / broadcasters / televisión móvil / televisión de alta definición.

\section{Digital Television deployment in Peru: A technological change without public politics}

SUMMARY: This article is about the opportunities and problems that brings the DTV (digital television) deployment in Peru. The main argument is that this technological change is also an opportunity for a structural change in peruvian's television. Therefore, this change must not focus in quality improvement only, but in the implementation of public politics that lead to an important change in television. The author suggests the following public politics for discussion: a) new operators join in, b) to dynamize the industry of contents, c) the deployment of DTV in local and regional television, d) to contribute with social inclusion by means of interactivity e) open signal television must be free, regardless medium (i.e: mobile phones), and

f) Running conditions according to each channel's business model.

Keywords: Digital television (DTV) / analogic shutdown / national television / public politics / broadcasters / mobile television / high definition TV (HDTV). 
T uego de que en abril del 2009 el ـerú eligiera el estándar japonésbrasileño de televisión digital terrestre (TDT), el gobierno conformó la Comisión Multisectorial Temporal Enargada de Formular Recomendaciones al Ministerio de Transportes y Comunicaciones (MTC) para la Elaboración del Plan Maestro de Implementación de la TDT. Sin considerar todos los aspectos involucrados en un proceso de implementación, el informe final presentado por dicha comisión en agosto del $2009^{1}$ ha centrado sus propuestas en cuatro ejes principales, los que permiten vislumbrar hacia dónde apunta el plan maestro que el MTC tendría que elaborar:

- Las propuestas planteadas se han realizado sobre la base "del respeto a los derechos adquiridos por los radiodifusores con autorización vigente".

- La formulación de un cronograma de simulcast ${ }^{2}$ y de apagón analógico por territorio (véase el cuadro).

Plazos de implementación de la TV digital en el Perú

\begin{tabular}{|c|c|c|c|c|c|}
\hline Territorio & Localidades & \multicolumn{2}{|c|}{ Población } & $\begin{array}{c}\text { Plazos para } \\
\text { iniciar simulcast }\end{array}$ & $\begin{array}{c}\text { Apagón } \\
\text { analógico }\end{array}$ \\
\hline 1 & Lima y Callao & 8,5 millones & $31 \%$ & 2010 al 2013 & 2020 \\
\hline 2 & $\begin{array}{l}\text { Arequipa, Cusco, Truji- } \\
\text { Ilo, Chiclayo, Piura, } \\
\text { Huancayo }\end{array}$ & 3,9 millones & $14 \%$ & 2010 al 2018 & 2025 \\
\hline 3 & $\begin{array}{l}\text { Chimbote, Ica, Iquitos, } \\
\text { Pucalpa, Juliaca, Puno, } \\
\text { Tacna, Ayacucho }\end{array}$ & 2,2 millones & $8 \%$ & 2010 al 2023 & 2030 \\
\hline 4 & Resto del país & 12,9 millones & $47 \%$ & a partir del 2010 & Sin plazo \\
\hline
\end{tabular}

Fuente: Informe de la Comisión Multisectorial Temporal Encargada de Formular Recomendaciones al Ministerio de Transportes y Comunicaciones para la Elaboración del Plan Maestro de Implementación de la TDT, agosto del 2009.

Elaboración propia.

1 Véase el informe completo en <http://www.mtc.gob.pe/portal/tdt/Documentos.pdf $>$.

2 A partir del 2010, los radiodifusores pueden solicitar la transmisión en simultáneo en analógico y digital. Vencido el plazo en simulcast, las frecuencias que queden disponibles solo se entregarán para transmisión digital. Con lo cual queda en evidencia que los radiodifusores actuales tienen amplias ventajas no solo para ingresar al campo digital, sino también para consolidarse en este mercado. 
- La recomendación de un programa de incentivos tributarios a los radiodifusores.

- El planteamiento de una campaña de comunicación para difundir las bondades de la TDT.

Transcurridos varios meses, y sin que haya indicios de aquel plan maestro, el viceministro de Comunicaciones Jorge Cuba ha dado a conocer, a fines de enero de este año, que el 30 de marzo del 2010 el canal estatal TV Perú iniciará las transmisiones de televisión digital en el país. Posteriormente, en este mismo año, se sumarán las estaciones ATV (9), Frecuencia Latina (2) y América Televisión (4), para transmitir los mundiales de fútbol, vóley y el popular programa $\mathrm{Al}$ fondo hay sitio, respectivamente. Hay especial apremio por iniciar dichas transmisiones por interés político, de un lado, y por mostrar los mejores productos de cada canal para insertarse en el escenario digital, del otro. Recordemos que el actual y el próximo son años electorales y no es mal negocio tender y tapizar puentes con los canales de televisión que ansían de una vez por todas "poner una pica en Flandes" en el escenario digital. Además, el 2010 es también un año mundialista y aunque hace 28 años el Perú no participa de una copa mundial, seguimos siendo un país ilusamente futbolero, razón más que suficiente para que ATV, que forma parte de la red de canales que el "fantasma" Gonzales ostenta en la región, transmita el mundial en televisión digital de alta definición. A todo ello se suman los anuncios comerciales de la inminente llegada de televisores digitales con el estándar japonés-brasileño ya incorporado, además de set of boxes para quienes aún no puedan cambiar de televisor. No puede dejarse de lado, en este escenario, el natural furor de futboleros y aficionados a las últimas tecnologías para renovar o agiornar sus actuales televisores.

No deja de ser sintomático que la historia de los cambios tecnológicos en la televisión peruana esté asociada al fútbol: la adopción del estándar NTSC de televisión a color se vio acelerada para que los canales transmitan a todo color el mundial Argentina 78, y aunque no se realizó una buena elección del estándar - recordemos que las siglas NTSC del National Television System Committee fueron convertidas en siglas de Never Twice Same Color - por lo menos aquella vez el Perú jugó su penúltimo mundial de fútbol. Ahora, la implementación de la televisión digital en el país se acelera con miras al mundial Sudáfrica 2010; esta vez no se trata de una mala elección del estándar, pero es una pésima señal que iniciemos las transmisiones sin haber debatido mínimamente el uso que se le pueda dar a la televisión digital, tanto para renovar esta industria, como para acompañar los distintos procesos socioeconómicos y culturales del país. 
Si el advenimiento de la televisión digital al país solo va a servir para ver con mejor resolución los programas de siempre o los espectáculos deportivos que responden a fenómenos sociales que van más allá de la televisión, habría que preguntarse entonces qué importancia tiene para la sociedad en su conjunto este cambio tecnológico. Porque si bien el cambio hacia la tecnología digital es un devenir en todos los procesos de digitalización que han venido ocurriendo en diversos ámbitos - y que la industria televisiva de señal abierta en el mundo la considera como una manera de ser más competitiva ante el avance de la televisión de pago y de las múltiples plataformas de consumo audiovisual - no debería ser una oportunidad desperdiciada para propiciar el cambio en la estructura misma de la televisión peruana. $\mathrm{Y}$ es que se trata de dejar en claro que la simple migración hacia la televisión digital no producirá cambios fundamentales en una industria que estructuralmente no ha variado sustancialmente en las últimas décadas y que aún no acaba de recomponerse de la pauperización ética y de contenidos que alcanzó su cima en el régimen fujimontesinista de la década de 1990.

En efecto, tenemos por un lado, un espectador televisivo que se encuentra hoy frente a mayores opciones de entretenimiento e información tales como las redes sociales y otras opciones de internet, los dispositivos perso- nales, la propia televisión de pago y los celulares. Plataformas desde las que se puede acceder a diversos contenidos y que consumen una buena parte de las horas de ocio, especialmente del público juvenil, que, a escala mundial, ha venido disminuyendo su consumo televisivo justamente por esta mayor oferta de entretenimiento, pero también porque la televisión no ha sabido encontrar las estrategias creativas y de contenido que mantengan el nivel de consumo de antaño y mucho menos la fidelidad de la audiencia. Más aún cuando este nuevo espectador es mucho más activo, desarrolla estrategias de desprogramación y anda a la búsqueda de convertirse también en un productor de contenidos, lo que se conoce como un prosumidor (productor-consumidor); allí están los casos de YouTube y Facebook para graficar claramente estos procesos.

$\mathrm{Y}$, por otro lado, tenemos una televisión nacional anquilosada en el tiempo pero que espera que se produzca el cambio tecnológico sin que cambie el statu quo, lo que no contribuiría con modificar los problemas estructurales de nuestra televisión: broadcasters mercantilistas; empresas débiles financiera y jurídicamente; escaso valor agregado de la pantalla televisiva $\mathrm{y}$, por lo mismo, baja inversión publicitaria en comparación con índices de la región; marco legal permisivo; alto consumo de televisión, especialmente en los sectores popula- 
res y escasa exigencia de una mejor televisión por el conjunto de los actores sociales. En buena cuenta, un buen caldo de cultivo para que la televisión se corrompa ante el poder político, como con gran evidencia se puso de manifiesto en la década de 1990, y que, pese a ello o tal vez justamente por ello, ninguno de los sucesivos gobiernos de Paniagua, Toledo y García supieron o quisieron corregir. $\mathrm{Y}$ es que:

[...] los políticos saben que en países como el Perú se requiere tener a la televisión como un aliado, que cumple diversas funciones de acuerdo al contexto y la conveniencia del momento: propaganda del régimen, desinformación, generación de "cortinas de humo", minimización de los problemas sociales, ataque frontal a los enemigos, entre otros. Tenerlos como opositores supone correr el riesgo de que los canales de televisión hagan bien su tarea: informar, fiscalizar, formar opinión pública (Arias 2008).

En este contexto, la introducción de la televisión digital al país, sin claras políticas públicas que propicien un cambio real en la televisión, no solo puede convertirse en un simple maquillaje, sino que también podría agravar algunos de los problemas estructurales y generar otros.
Algunas de las principales líneas de políticas públicas de televisión que deberían discutirse en el proceso de implementación de la TDT serían las que se detallan a continuación. ${ }^{3}$

\section{Ingreso de nuevos operadores}

El Ministerio de Transportes y Comunicaciones, por medio de la Resolución Ministerial 645-2006-MTC establece que la Banda de 470-584 $\mathrm{MHz}$ (canales 14 al 32) queda reservada para el servicio de televisión digital terrestre. Son estas las nuevas licencias sobre las que deberá legislarse. ¿Se tienen que dar estas licencias a los actuales operadores de televisión? ¿Es necesario licitar estas nuevas licencias como si se tratara de un nuevo negocio? ¿Deberían los actuales operadores tener derecho de asignación automática una licencia en el campo digital? Son estas las preguntas que deben ser absueltas en el proceso de implementación y cuya respuesta puede variar el actual panorama televisivo. En todo caso, conviene tomar en cuenta las alternativas manejadas en la experiencia de otros países (Godoy 2005):

- Licitación pública de las nuevas licencias. Las nuevas bandas de televisión digitales son consideradas un servicio nuevo que no tendría que asignarse directamente a los

3 Parte de estos lineamientos fueron desarrollados en el artículo "Hacia la formulación de políticas públicas para el cambio digital" (Arias 2009). 
operadores de televisión analógica (Francia, Inglaterra).

- Régimen de licencias canal por canal en vez de entregar todo el ancho de banda $(6 \mathrm{MHz})$ en bloque a los concesionarios a efectos de que emitan varios contenidos (Suecia, Finlandia).

- Concesiones nacionales, regionales y locales por separado (España).

La apertura del sistema a nuevos operadores puede contribuir a democratizar e insuflar de una mayor pluralidad a la televisión peruana, a condición, claro está, de que se establezcan unas reglas claras y transparentes en esta asignación de licencias que debería ser entregada por una instancia independiente y no por el MTC.

Nuevos operadores que podrían tener fines comerciales, culturales, educativos, políticos, religiosos, entre otros. Y es que no se trata solamente de que la señal abierta sea tomada por empresarios que tienen intereses comerciales, sino también por organizaciones e instituciones que tienen algo que aportar al interés público, que es la esencia de la televisión de señal abierta.

Y claro está que habría que poner en cuestión esos "derechos adquiridos" que reclaman nuestros broadcasters y que desde el MTC se viene considerando de acuerdo al informe de la comisión temporal señalada líneas arriba. No solo porque el escenario digital es técnicamente otro negocio que se desarrolla en otra banda del espectro radioeléctrico, sino también, y sobre todo, porque esos derechos habrían sido adquiridos con la performance que los canales han tenido durante la operación de sus licencias. Y ya sabemos la televisión que hacen, y ya sabemos la televisión que hicieron en la década de 1990. Con todo este accionar, si el Estado se empeña en "respetar los derechos adquiridos" de los broacasters sin más condición, no solo les entregaría una licencia en el escenario digital, sino también una licencia para que estos sigan "operando" como han venido haciéndolo.

\section{Dinamizar la industria televisiva pero también la de contenidos}

Además, licitar públicamente las licencias para posibilitar el ingreso de nuevos operadores podría dinamizar la industria televisiva, tanto porque habría una mayor competencia como porque se requeriría de una mayor oferta de contenidos para atender las necesidades de programación de los nuevos canales. Y como consecuencia de ello, también se dinamizaría la industria de contenidos que, pese al crecimiento económico del país, ha venido trabajando con limitados presupuestos, por debajo de los costos de hace más de diez años, en gran medida porque en la práctica solo tres canales nacionales están en capacidad de acoger producciones independientes.

Con contados puertos de salida en el mercado local, con pocas posibilida- 
des reales de exportación a los mercados más importantes, dados los escasos valores de producción por la baja inversión, la producción televisiva peruana se ve envuelta en un círculo vicioso del que no ha podido escapar. De allí la importancia de abrir el sistema y, siendo más audaces, generar un marco legal que propicie que los canales de televisión se dediquen solo a programar, seleccionando para ello los mejores contenidos locales e importados. De este modo, la producción independiente tendría más posibilidades de colocar sus productos en alguna de las pantallas televisivas, incrementando valores de producción por una mayor inversión presupuestal al reducirse el poder negociador de unos pocos canales. Todo ello con los objetivos de desarrollar unos contenidos que ofrezcan nuevas perspectivas e innovaciones en los diversos géneros, pero también de ir construyendo un estilo de hacer televisión reconocible y valorable en el mercado internacional.

\section{Televisoras regionales y locales}

La televisión peruana es centralista, hecha por y desde los criterios y prioridades de Lima. Y aunque es verdad que en las diversas regiones del país van apareciendo empresas que pugnan por desarrollar una televisora local, más impulsados por intereses económicos y políticos antes que de aporte a su comunidad - lo que no se contradice con que tengan que dar cuenta de los elementos locales para interesar a su audiencia-, no contamos con sólidos canales regionales o locales que contrarresten la hegemonía de las cadenas nacionales.

Si el país quiere tomar en serio el proceso de regionalización no puede dejar de lado que junto con el desarrollo económico de cada ciudad tiene que haber un mayor desarrollo cultural y comunicacional que acompañe todo el proceso.

Por este motivo, la cooperación económica y técnica ofrecida por el estándar de TDT japonés-brasileño debería destinarse, en primer lugar, a posibilitar que efectivamente las televisoras regionales y locales puedan migrar a la televisión digital, a condición de presentar unos proyectos que hagan posible su consolidación y su sostenibilidad en el tiempo. Este es un tema fundamental detrás del cual estarán las cadenas nacionales, pero que una clara política de lo que se debe hacer en este campo debería ser el freno para el habitual apetito voraz de las canales nacionales, que, ya sabemos, buscan generar ganancias privadas y deudas públicas, con el matiz, en este caso, de lograr inversión pública para el lucro privado.

\section{Interactividad ¿̇para qué fines?}

Una de las posibilidades que trae consigo la televisión digital es la interactividad. Más allá de aspectos técnicos, que deberán cotejarse en su momento, como los decodificadores más convenientes para cumplir con esta función, 
habrá que interrogarse sobre qué servicios interactivos queremos que se implementen en la televisión. Librados a su suerte, los canales de televisión bien podrían generar servicios interactivos con fines estrictamente comerciales, que permitan al televidente participar de programas concurso o interactuar con algún mensaje publicitario que lo conduzca a una posible compra (pasaríamos rápidamente del "¡llame ya!" al "¡compre ya!" con solo el control remoto).

La interactividad en la televisión, sin embargo, puede cumplir funciones sociales, desde ofrecer verdaderamente más información sobre la noticia que se está viendo en directo hasta gestionar citas médicas o trámites gubernamentales, pasando por el voto electrónico en las diversas elecciones.

Claro que bien aprovechada la interactividad puede posibilitar el desarrollo de contenidos lúdicos y creativos y ciertamente lucrativos, pero no deberíamos desaprovechar esta posibilidad para reducir la brecha digital $\mathrm{y}$, en suma, para contribuir con la inclusión social, tomando en cuenta las grandes poblaciones a las que no llega el Estado. Y esta posibilidad no debería estar librada al juego del mercado sino convertirse, con los afinamientos del caso, en una política pública de televisión.

\section{Televisión móvil żgratis?}

El celular se ha convertido en el dispositivo personal de mayor desarrollo y penetración en los últimos años. A las funciones básicas de hacer llamadas y enviar mensajes de texto, se han incorporado una diversidad de opciones que hacen que este dispositivo se convierta en un verdadero centro de comunicación, información y entretenimiento. La posibilidad de ver televisión en algunos modelos de celulares ya se ha demostrado y puesto en práctica en diversos países.

Si bien nuestro parque de celulares no tiene la sofisticación que ostentan otros mercados más desarrollados, es importante notar el grado de penetración alcanzado (casi $80 \%$ ) y la pronta migración hacia equipos multimedia. La gran población joven del país hace prever un cada vez más acelerado crecimiento, tanto de la tenencia de equipos como en los usos y servicios que se desplegarán con este dispositivo. De allí la importancia de contemplar los celulares como un nuevo canal de distribución de televisión. Si la televisión estaba pasiva en los hogares esperando la llegada de sus miembros, hoy en día debe salir a su búsqueda y la más clara y eficaz alternativa es el celular.

Hasta hoy se había considerado que la televisión de señal abierta es gratuita, y así la recibimos en los hogares. Empresas de telecomunicaciones y canales de televisión ven en la transmisión televisiva a través de celular una oportunidad de negocio. Sin embargo, no deberíamos perder de vista, bajo ningún concepto, que la te- 
levisión de señal abierta debe ser gratuita, más allá del dispositivo en que se reciba. El negocio de los canales y operadores de telecomunicaciones tendría que desarrollarse en otra dirección, tanto por la publicidad inserta en los programas como por las posibilidades de más tráfico telefónico o de mensajes de texto a efectos de participar en ciertos programas televisivos, sin dejar de lado todas las posibilidades de crear contenidos audiovisuales específicos para celulares, como es el caso de los moviesodios. Otra cosa es que los operadores de telecomunicaciones lleguen a acuerdos comerciales con canales de pago para transmitir algunos de sus contenidos, en cuyo caso se entiende que sería un servicio pagado por el abonado de telefonía.

\section{Alta definición o televisión digital estándar}

Otro eje de discusión lo puede constituir la opción de televisión de alta definición versus la televisión digital estándar, opción esta última que posibilita que por un mismo ancho de banda se transmitan más contenidos.

La apuesta por la televisión de alta definición supone una mayor inversión en valores de producción, que tiene sentido en ciertas producciones especiales como las películas, los musicales o los programas deportivos. Lo que deberíamos evitar es que se utilice el argumento de la necesidad de hacer televisión de alta definición para evitar el ingreso de otros operadores que bien podrían compartir parte del ancho de banda con el que se opera en el país (6Mhz), si es que un canal no está en condiciones reales de desarrollar nuevos contenidos.

Como se ha señalado en diversos estudios, no existe una real dicotomía entre la modalidad de alta definición y la opción por el multicasting, puesto que uno de los atributos de los estándares es la flexibilidad, que permite que cada operador configure sus transmisiones de acuerdo con su plan de negocios (CNTV y PUC de Chile 2006).

Por estos motivos, la entrega de licencias en el escenario digital debería estar acompañada de mínimas condiciones de explotación, de acuerdo con los modelos de negocio que cada canal pretende poner en marcha.

$\mathrm{Si}$ un canal espera desarrollar diversos contenidos para usar todo el ancho de banda, habría que cuidar que no ocurra lo que sucede hoy en la señal abierta, donde un canal dedica gran parte de su programación a repetir programas de hace más de veinte años y a emitir películas orientales de escaso interés e ínfimos valores de producción (imaginemos lo que haría este canal si tuviera todo el ancho de banda para desarrollar cuatro o cinco contenidos).

Además, aunque no podría legislarse al respecto, habría que estar vigilantes para que los canales no empiecen a transmitir en alta definición los mismos programas de humor, chis- 
me, tragedia informativa o conversación simple, sin que se haya invertido en mejorar su calidad. De lo contrario, estaríamos viendo la misma televisión pero más bonita. Todo un contrasentido.

Finalmente, es necesario dejar en claro que plantear la necesidad de que se establezcan políticas públicas para la implementación de la televisión digital no es una vuelta al pasado a un modelo estatista y regulador en un escenario de economía neoliberal. Menos cuando este último modelo hace rato que ha dejado notar las enormes distorsiones que puede generar sin una clara posición política de la labor que le compete al Estado en una economía de libre mercado. Es simple y llanamente hacer que el Estado y el conjunto de actores sociales consideren y evalúen el impacto que puede tener la tecnología digital en el desarrollo de las industrias audiovisuales; la posibilidad de mejorar el rol que cumple la televisión en el país; la posibilidad de utilizar la tecnología digital para democratizar las comunicaciones; el modo en que digitalmente se puede acercar el Estado a la población; la opción de reducir la brecha digital; la necesidad de acompañar los procesos de regionalización; $y$, en suma, la imperiosa necesidad de hacer de este un país más inclusivo.

En otras palabras, la implementación de la TDT en el país no puede reducirse a generar cronogramas y pla- zos de apagón analógico, si antes no se define el bien público que está en juego y se toman las decisiones que se traduzcan en claros lineamientos para que el conjunto de actores sociales opere no solo en función de sus intereses particulares, sino también en concordancia con los intereses del conjunto del país.

\section{Bibliografía}

Arias Carbajal, Gerardo (abril del 2009). "Hacia la formulación de políticas públicas para el cambio digital". La Mirada de Telemo 2. Lima: Pontificia Universidad Católica del Perú.

_ (2008). "Practically soap operas, practically series: Peruvians miniseries as an escape from TV fiction", en VASSALlo, María Inmacolata y Lorenzo VILCHES (coords.). Global markets, local stories. Obitel Yearbook 2008. São Paulo: Globo Universidade.

Consejo Nacional de Televisión de Chile (2006). "Situación de la televisión digital en el mundo e implicancias para Chile".

Godoy EtcheverRy, Sergio (noviembre del 2005). "Desafíos regulatorios para la adopción de la televisión digital terrestre en Chile". Documento elaborado para el Consejo Nacional de Televisión de Chile. Santiago.

Gómez Germano, Gustavo (2007). La radio y la televisión en la era digital. 
Bogotá: Centro de Competencia en Comunicación para América Latina/Fiedrich Ebert Stiftung.

Informe de la Comisión Multisectorial Temporal Encargada de Formular Recomendaciones al Ministerio de Transportes y Comunicaciones para la Elaboración del Plan Maestro de Implementación de la TDT [en línea]. www.mtc.gob.pe/portal/inicio.html>. [Consulta: 15 de diciembre del 2009.]

Pontificia Universidad Católica de Chile (2006). Análisis de los estándares de transmisión de televisión digital terrestre y su aplicabilidad al medio nacional. Santiago: Escuela de Ingeniería. 